\title{
Radon-Nikodým Theorems for Finitely Additive Multimeasures
}

\author{
Luisa Di Piazza and Giovanni Porcello
}

\begin{abstract}
In this paper we deal with interval multimeasures. We show some RadonNikodým theorems for such multimeasures using multivalued Henstock or HenstockKurzweil-Pettis derivatives. We do not use the separability assumption in the results.
\end{abstract}

Keywords. Multifunction, Henstock-Kurzweil integral, Henstock-Kurzweil-Pettis integral, selection, Radon-Nikodým theorem

Mathematics Subject Classification (2010). Primary 26A39, 28B20, 46G10, secondary 54C60, 54C65

\section{Introduction}

One of the most fascinating problems arising when we deal with multimeasures is the represention of a multimeasure as an integral, i.e. the existence of a Radon-Nikodým derivative.

Several papers concerning this question appeared since the 1970's where pioneering results have been established amongst others by Artstein [1], Costé [6], Costé and Pallu de la Barrière [7]. These papers deal with countably additive multimeasures and use classical notions of integral existing in literature.

In the 1990's other results dealing with finitely additive multimeasures have been obtained by A. Martellotti, K. Musiał and A. R. Sambucini (see $[16,17,20])$. In particular, they have been extended the study beyond the Banach spaces (in particular, to locally convex spaces), but also in this case classical integrals are used for the representation.

In general the results existing in literature use multimeasures defined on a $\sigma$-algebra. Moreover, most of them uses the separability assumption.

L. Di Piazza, G. Porcello: Department of Mathematics and Computer Sciences, University of Palermo, via Archirafi 34, 90123 Palermo, Italy;

dipiazza@math.unipa.it; porcello@math.unipa.it

The authors were partially supported by the Polish Ministry of Higher Education, Grant No. NN201 416139 
In this paper we deal with the Radon-Nikodým problem for multimeasures defined on the family $\mathcal{I}$ of all non trivial closed subintervals of $[0,1]$ and consequently we look for Radon-Nikodým derivatives of Henstock type. This is the first paper where that problem has been undertaken.

The Henstock integral has been introduced in the 1960's independently by Henstock [12] and Kurzweil [15], by a simple modification of Riemann's method. It is a non absolutely convergent integral more general than Lebesgue's one, integrates all derivatives and its primitive is a finitely additive interval function.

Our starting point is the remarkable recent article of B. Cascales, V. Kadets and J. Rodríguez [5], where they obtain two Radon-Nikodým theorems for countably additive multimeasures without any separability assumption.

Here we go on in such kind of investigation and we consider finitely additive multimeasures defined on $\mathcal{I}$, taking convex compact values or convex weakly compact values, in an arbitrary Banach space $X$.

The paper is organized as follows. In Section 2 we give necessary notations, definitions and preliminaries.

In Section 3 we extend to the multivalued case the notion of variational measure already known for vector valued interval measure. This measure is a useful tool for our investigation.

In Section 4 we prove the main results. In the convex compact case we obtain a Radon-Nikodým theorem for dominated interval multimeasures (see Theorem 4.2) that improves [5, Theorem 3.1]. To get our goal we use an extension of a finitely additive multimeasure to a countably additive multimeasure defined in the $\sigma$-algebra of the Borel subsets of $[0,1]$ (see Proposition 4.1).

In the more general context of convex weakly compact valued multimeasures we find an HKP-integrable derivative under the hypothesis of absolute continuity for the associated variational measure (see Theorem 4.6). Also in such a case we do not require the separability of the target Banach space $X$, but we assume that $X$ possesses the Radon-Nikodým property (shortly RNP).

\section{Notations and preliminary definitions}

Let $[0,1]$ be the unit interval of the real line, endowed with the usual topology and the Lebesgue measure $\lambda$. We denote by $\mathcal{L}$ the family of all measurable subsets of $[0,1]$, by $\mathcal{A}$ and $\sigma(\mathcal{A})$ respectively the ring and the $\sigma$-ring generated by the subintervals $[a, b) \subseteq[0,1]$ and by $\mathcal{I}$ the family of all non trivial subintervals of $[0,1]$.

A partition in $[0,1]$ is a finite collection of pairs $\left\{\left(I_{j}, t_{j}\right)\right\}_{j=1}^{q}$, where $I_{1}, \ldots, I_{q}$ are non-overlapping subintervals of $[0,1]$ and $t_{1}, \ldots, t_{q} \in[0,1]$. Given a subset $E$ of $[0,1]$, we say that the partition $\left\{\left(I_{j}, t_{j}\right)\right\}_{j=1}^{q}$ is anchored on $E$ if $t_{j} \in E$ for each $j=1, \ldots, q$. If $\bigcup_{j=1}^{q} I_{j}=[0,1]$, we say that $\left\{\left(I_{j}, t_{j}\right)\right\}_{j=1}^{q}$ is a partition of $[0,1]$. 
A gauge on $B \subset[0,1]$ is a positive function on $B$. Given a gauge $\delta$, we say that a partition $\left\{\left(I_{j}, t_{j}\right)\right\}_{j=1}^{q}$ is $\delta$-fine if $I_{j} \subset\left(t_{j}-\delta\left(t_{j}\right), t_{j}+\delta\left(t_{j}\right)\right)$ for every $j=1, \ldots, q$.

Let denote by $X$ a real Banach space, with dual $X^{*}$. The closed unit ball of $X\left(\operatorname{resp} . X^{*}\right)$ is denoted by $B(X)\left(\operatorname{resp} . B\left(X^{*}\right)\right)$.

Definition 2.1. A function $f:[0,1] \rightarrow X$ is said to be Henstock integrable (or simply $H$-integrable) on $[0,1]$ if there exists $x \in X$ with the following property: for every $\varepsilon>0$ there exists a gauge $\delta$ on $[0,1]$ such that

$$
\left\|\left|\sum_{j=1}^{q} f\left(t_{j}\right)\right| I_{j} \mid-x\right\|<\varepsilon,
$$

for every $\delta$-fine partition $\left\{\left(I_{j}, t_{j}\right)\right\}_{j=1}^{q}$ of $[0,1]$.

We call $x$ the Henstock integral of $f$ on $[0,1]$ and we set $(H) \int_{0}^{1} f d \lambda:=x$.

It is well known that if $f:[0,1] \rightarrow X$ is Henstock integrable on $[0,1]$ and $I \in \mathcal{I}$, also the function $f \chi_{I}$ is Henstock integrable on $[0,1]$ [21, Theorem 3.3.4]. We say in such a case that $f$ is Henstock integrable on $I$. If $X=\mathbb{R}$, then $f$ is said to be Henstock-Kurzweil integrable or simply HK-integrable on [0,1] and we denote by $(H K) \int_{0}^{1} f d \lambda$ the corresponding interval.

Definition 2.2. A function $f:[0,1] \rightarrow X$ is said to be scalarly HK-integrable (resp. scalarly integrable) if for every $x^{*} \in X^{*}$ the real function $\left\langle x^{*}, f(\cdot)\right\rangle$ is $H K$-integrable (resp. integrable).

A scalarly $H K$-integrable (resp. scalarly integrable) function $f:[0,1] \rightarrow X$ is said to be Henstock-Kurzweil-Pettis integrable or simply HKP-integrable (resp. Pettis integrable) on $[0,1]$ if for every interval $I \in \mathcal{I}$ (resp. for every $E \in \mathcal{L}$ ), there exists $x_{I} \in X\left(\operatorname{resp} . x_{E} \in X\right)$ such that

$$
\begin{gathered}
\left\langle x^{*}, x_{I}\right\rangle=(H K) \int_{I}\left\langle x^{*}, f\right\rangle d \lambda, \quad \text { for every } x^{*} \in X^{*} \\
\left(\operatorname{resp.}\left\langle x^{*}, x_{E}\right\rangle=\int_{E}\left\langle x^{*}, f\right\rangle d \lambda, \quad \text { for every } x^{*} \in X^{*}\right) .
\end{gathered}
$$

We call $x_{I}$ (resp. $x_{E}$ ) the HKP-integral of $f$ on $I$ (resp. Pettis integral of $f$ on $E)$ and we write $(H K P) \int_{I} f d \lambda:=x_{I}\left(\operatorname{resp} .(P) \int_{E} f d \lambda:=x_{E}\right)$.

For more details about vector valued functions see $[8,18,21]$.

The class of all non-empty subsets of $X$ is $2^{X}$. By $c l(X), c c(X), c b c(X)$, $c k(X), c w k(X)$ we denote respectively the subfamilies of $2^{X}$ of all closed, closed convex, closed convex bounded, convex compact and convex weakly compact subsets of $X$. 
On $2^{X}$ we consider the Minkowski addition

$$
C+C^{\prime}:=\overline{\left\{x+x^{\prime}: x \in C, x^{\prime} \in C^{\prime}\right\}}
$$

and the standard multiplication by scalars.

For every $C \in 2^{X}$, the support function of $C$ is denoted by $s(\cdot, C)$ and defined on $X^{*}$ by $s\left(x^{*}, C\right):=\sup \left\{\left\langle x^{*}, x\right\rangle: x \in C\right\}$, for each $x^{*} \in X^{*}$.

We denote by $d_{H}$ the Hausdorff distance in $2^{X}$ :

$$
d_{H}\left(C, C^{\prime}\right):=\max \left\{e\left(C, C^{\prime}\right), e\left(C^{\prime}, C\right)\right\}, C, C^{\prime} \in 2^{X},
$$

where $e\left(C, C^{\prime}\right)=\sup \left\{d\left(x, C^{\prime}\right): x \in C\right\}$ is the excess of $C$ over $C^{\prime}$, while $d(x, C)=\inf \{\|x-y\|: y \in C\}$ is the distance of $x$ from $C$. For $A \in 2^{X}$, we set $\|A\|:=\sup \{\|x\|: x \in A\}$ and we call it the radius of $A$.

A map $F:[0,1] \rightarrow \operatorname{cl}(X)$ is called a multifunction. A function $f:[0,1] \rightarrow X$ is called a selection of $F$ if for every $t \in[0,1]$ one has $f(t) \in F(t)$.

A multifunction $F$ is said to be scalarly integrable (resp. scalarly $H K$ integrable) if for every $x^{*} \in X^{*}$ the map $s\left(x^{*}, F(\cdot)\right)$ is integrable (resp. $H K$ integrable).

More information concerning Pettis integrability of multifunctions can be found in [19].

Definition 2.3. A multifunction $F:[0,1] \rightarrow c b c(X)(c k(X), c w k(X))$ is said to be Henstock-Kurzweil-Pettis integrable or simply HKP-integrable (resp. Pettis integrable) in $c b c(X)(c k(X), c w k(X))$ if $F$ is scalarly $H K$-integrable (resp. scalarly integrable) and for every interval $I \in \mathcal{I}$ (resp. for every $E \in \mathcal{L}$ ), there exists $C_{I} \in \operatorname{cbc}(X)(c k(X), c w k(X))$ (resp. $\left.C_{E} \in \operatorname{cbc}(X)(c k(X), c w k(X))\right)$ such that

$$
\begin{gathered}
s\left(x^{*}, C_{I}\right)=(H K) \int_{I} s\left(x^{*}, F\right) d \lambda, \quad \text { for every } x^{*} \in X^{*} \\
\left(\text { resp. } s\left(x^{*}, C_{E}\right)=\int_{E} s\left(x^{*}, F\right) d \lambda, \quad \text { for every } x^{*} \in X^{*}\right) .
\end{gathered}
$$

We call $C_{I}$ (resp. $C_{E}$ ) the HKP-integral of $F$ over $I$ (resp. Pettis integral of $F$ over $E$ ) and we set $(H K P) \int_{I} F d \lambda:=C_{I}$ (resp. $\left.(P) \int_{E} F d \lambda:=C_{E}\right)$.

More information concerning Pettis integrability of multifunctions can be found in [19].

Definition 2.4. A multifunction $F:[0,1] \rightarrow \operatorname{cbc}(X)(\operatorname{ck}(X), c w k(X))$ is said to be Henstock integrable if there exists $W \in \operatorname{cbc}(X)(c k(X), c w k(X))$ with the following property: for every $\varepsilon>0$ there exists a gauge $\delta$ on $[0,1]$ such that for every $\delta$-fine partition $\left\{\left(I_{j}, t_{j}\right)\right\}_{j=1}^{p}$ of $[0,1]$ we have

$$
d_{H}\left(W, \sum_{j=1}^{p} F\left(t_{j}\right)\left|I_{j}\right|\right)<\varepsilon .
$$

$W$ is called the Henstock integral of $F$ and we write $(H) \int_{I} F d \lambda:=W$. 
A multifunction $M: \mathcal{L} \rightarrow \operatorname{cl}(X)$ is said to be a $d_{H}$-multimeasure if for every sequence $\left(A_{n}\right)_{n \geq 1} \subset \mathcal{L}$ of pairwise disjoint sets with $A=\bigcup_{n \geq 1} A_{n}$, we have $d_{H}\left(M(A), \sum_{k=1}^{n} M\left(A_{k}\right)\right) \rightarrow 0$ as $n \rightarrow+\infty$.

A multifunction $M: \mathcal{L} \rightarrow \operatorname{cl}(X)$ is said to be a weak multimeasure or simply a multimeasure if for every $x^{*} \in X^{*}$, the map $A \mapsto s\left(x^{*}, M(A)\right)$ is a real valued measure.

It is known that every $\mathrm{cl}(X)$-valued $d_{H}$-multimeasure is a multimeasure (see [14, Proposition 8.4.7]).

The two notions coincide whenever the multimeasure takes its values in $c w k(X)$ (see [14, Theorem 8.4.10]).

We say that the multimeasure $M: \mathcal{L} \rightarrow 2^{X}$ is $\lambda$-continuous and we write $M \ll \lambda$, if $\lambda(A)=0$ yields $M(A)=\{0\}$.

Given a multimeasure $M: \mathcal{L} \rightarrow 2^{X}$, a vector measure $m: \Sigma \rightarrow X$ such that $m(A) \in M(A)$ for every $A \in \mathcal{L}$ is called a selection of $M$.

Moreover, the variation of $M$ is the extended non-negative function $|M|$ whose value on a set $A \in \mathcal{L}$ is given by

$$
|M|(A):=\sup \sum_{i}\left\|M\left(A_{i}\right)\right\|
$$

where the supremum is taken over all finite partitions $\left(A_{i}\right)_{i}$ of $A$ in $\mathcal{L}$.

If $|M|([0,1])<+\infty$, then $M$ is called of finite variation. If there exists a sequence $\left(A_{n}\right)_{n} \subset \mathcal{L}$ of pairwise disjoint sets covering $[0,1]$ and such that $|M|\left(A_{n}\right)<+\infty$ for every $n$, then $M$ is called of $\sigma$-finite variation.

\section{Interval multimeasures and their selections. Varia- tional measures}

We start with the following definitions.

Definition 3.1. An interval multifunction $\Phi: \mathcal{I} \rightarrow c w k(X)$ is said to be finitely additive if for every non-overlapping intervals $I_{1}, I_{2} \in \mathcal{I}$ such that $I_{1} \cup I_{2} \in \mathcal{I}$ we have $\Phi\left(I_{1} \cup I_{2}\right)=\Phi\left(I_{1}\right)+\Phi\left(I_{2}\right)$.

Remark 3.2. The primitives of Henstock or Henstock-Kurzweil-Pettis integrable multifunctions are interval multimeasures. Moreover, it is known that if $F:[0,1] \rightarrow c w k(X)$ is Pettis integrable in $c w k(X)$, then its primitive is $\sigma$-additive (see [18]). If we set $\Phi(I):=\nu(I), I \in \mathcal{I}$, then $\Phi$ is an interval multimeasure.

Definition 3.3. A multifunction $\Psi: \mathcal{A} \rightarrow c w k(X)$ is said to be a finitely additive multimeasure if for every $A_{1}, A_{2} \in \mathcal{A}$ whose interiors are disjoint we have $\Psi\left(A_{1} \cup A_{2}\right)=\Psi\left(A_{1}\right)+\Psi\left(A_{2}\right)$. 
Remark 3.4. In the following we identify a finitely additive interval multifunction $\Phi: \mathcal{I} \rightarrow c w k(X)$ with the finitely additive multimeasure $\Psi: \mathcal{A} \rightarrow c w k(X)$ defined by $\Psi(A):=\sum_{j=1}^{q} \Phi\left(I_{j}\right)$, where $A=\bigcup_{j=1}^{q} I_{j}$ and $I_{1}, \ldots, I_{q}$ are pairwise disjoint subintervals of $[0,1]$. We use a similar identification for the corresponding selections.

Hence we call interval multimeasure every finitely additive interval multifunction and interval measure every finitely additive interval function.

Moreover, we observe that if $\Phi: \mathcal{I} \rightarrow c w k(X)$ is an interval multimeasure, then for every $x^{*} \in X^{*}, s\left(x^{*}, \Phi(\cdot)\right)$ is a real-valued interval measure.

An interval measure $\phi: \mathcal{I} \rightarrow X$ is said to be a selection of an interval multimeasure $\Phi$ if $\phi(I) \in \Phi(I)$ for every $I \in \mathcal{I}$.

We recall that for $\emptyset \neq K \subset X$, an element $x \in K$ is called an exposed point of $K$ if there exists $x^{*} \in X^{*}$ such that $\left\langle x^{*}, x\right\rangle>\left\langle x^{*}, y\right\rangle$ for every $y \in K \backslash\{x\}$; an exposed point $x$ of $K$ is called a strongly exposed point of $K$ if for every $\left(x_{n}\right)_{n} \subset K$ with $\left\langle x^{*}, x_{n}\right\rangle \rightarrow\left\langle x^{*}, x\right\rangle$, then $\left\|x_{n}-x\right\| \rightarrow 0$, where $x^{*}$ is the functional that exposes $x$. We denote by $\exp (K)$ (resp. $\operatorname{str} \exp (K))$ the set of the exposed points (resp. strongly exposed points) of $K$.

It is known that if $K \in c k(X)$ (resp. $K \in c w k(X))$, then $\exp (K) \neq \emptyset$ $($ resp. $\operatorname{str} \exp (K) \neq \emptyset)$ and $K=\overline{c o}(\exp (K))($ resp. $K=\overline{c o}(\operatorname{str} \exp (K)))$ (see [4, Theorem 3.2.2] and [4, Theorem 3.6.1]).

The following result is well known in case of countably additive multimeasures (see [13]). We omit its proof, since it is very similar to that in [13, Proposition 2.1].

Proposition 3.5. Let $\Phi: \mathcal{I} \rightarrow c k(X)$ be an interval multimeasure. If $x_{0} \in \exp (\Phi([0,1]))$ then there exists a selection $\phi: \mathcal{I} \rightarrow X$ of $\Phi$ such that $\phi([0,1])=x_{0}$ and $\phi(I) \in \exp (\Phi(I))$ for every $I \in \mathcal{I}$.

With similar argument, we obtain

Proposition 3.6. Let $\Phi: \mathcal{I} \rightarrow c w k(X)$ be an interval multimeasure. If $x_{0} \in \operatorname{str} \exp (\Phi([0,1]))$ then there exists a selection $\phi: \mathcal{I} \rightarrow X$ of $\Phi$ such that $\phi([0,1])=x_{0}$ and $\phi(I) \in \operatorname{str} \exp (\Phi(I))$ for every $I \in \mathcal{I}$.

Now we extend the notion of variational measure to additive interval multimeasures (cf. [2] or [9]). This notion is a useful tool to study the primitives of real valued or, more in general, vector valued integrable functions.

Given an interval multimeasure $\Phi: \mathcal{I} \rightarrow c w k(X)$, a gauge $\delta$ and a set $E \subset[0,1]$, we define

$$
\operatorname{Var}(\Phi, \delta, E)=\sup \sum_{j=1}^{p}\left\|\Phi\left(I_{j}\right)\right\|
$$


where the supremum is taken over all the $\delta$-fine partitions $\left\{\left(I_{j}, t_{j}\right)\right\}_{j=1}^{p}$ anchored on $E$. Then we set

$$
V_{\Phi}(E):=\inf \{\operatorname{Var}(\Phi, \delta, E): \delta \text { gauge on } E\}
$$

$V_{\Phi}$ is called the variational measure generated by $\Phi$.

Remark 3.7. If $\Phi$ is an interval multimeasure, then $V_{\Phi}$ is the variational measure generated by the single valued map $R \circ \Phi$, where $R: c w k(X) \rightarrow l_{\infty}\left(B\left(X^{*}\right)\right)$ is the Rådstrom Embedding defined by $R(C):=s(\cdot, C)$, for $C \in c w k(X)$. In fact, for $I \in \mathcal{I}$ we obtain:

$$
\begin{aligned}
\|R(\Phi(I))\|_{l_{\infty}} & =\|s(\cdot, \Phi(I))\|_{l_{\infty}} \\
& =\sup _{x^{*} \in B\left(X^{*}\right)}\left|s\left(x^{*}, \Phi(I)\right)\right| \\
& =\sup _{x^{*} \in B\left(X^{*}\right)}\left|s\left(x^{*}, \Phi(I)\right)-s\left(x^{*},\{0\}\right)\right| \\
& =d_{H}(\Phi(I),\{0\}) \\
& =\|\Phi(I)\| .
\end{aligned}
$$

Consequently, $\operatorname{Var}(\Phi, \delta, E)=\operatorname{Var}(R(\Phi), \delta, E)$ for any gauge $\delta$ and any set $E \subset[0,1]$, and $V_{\Phi}(E)=V_{R \circ \Phi}(E)$ for any set $E \subset[0,1]$.

Therefore, as in the $X$-valued case, $V_{\Phi}$ is a metric outer measure on $[0,1]$ (see [2]) and a measure over all Borel sets of $[0,1]$.

We say that the variational measure $V_{\Phi}$ is $\sigma$-finite if there exists a sequence of (pairwise disjoint) sets $\left(E_{n}\right)_{n \geq 1}$ covering $[0,1]$ and such that $V_{\Phi}\left(E_{n}\right)<\infty$, for every $n \geq 1$. Moreover, we say that $V_{\Phi}$ is absolutely continuous with respect to $\lambda$ (or $\lambda$-continuous) and we write $V_{\Phi} \ll \lambda$ if for every $E \in \mathcal{L}$ with $\lambda(E)=0$ we have $V_{\Phi}(E)=0$.

Remark 3.8. Taking into account that $V_{\Phi}=V_{R \circ \Phi}$ and using [2, Corollary 2.3], we have $\sigma$-finiteness of every $\lambda$-continuous variational measure.

\section{Main results}

4.1. The $c k(X)$ case. We start by proving an extension result.

Proposition 4.1. Let $\Phi: \mathcal{I} \rightarrow c k(X)$ be an interval multimeasure such that there exists a set $Q \in \operatorname{ck}(X)$ with $\Phi(I) \subseteq|I| Q$ for every $I \in \mathcal{I}$.

Then $\Phi$ can be extended to a multimeasure $M: \sigma(\mathcal{A}) \rightarrow c k(X)$ such that $M(B) \subseteq \lambda(B) Q$ for every $B \in \sigma(\mathcal{A})$. 
Proof. We observe that for every $x^{*} \in X^{*}, s\left(x^{*}, \Phi\right)$ is a real-valued measure and $-s\left(-x^{*}, Q\right)|I| \leq s\left(x^{*}, \Phi(I)\right) \leq s\left(x^{*}, Q\right)|I|, \quad$ for every $I \in \mathcal{I}$.

Fix $x^{*} \in X^{*}$. Then $s\left(x^{*}, \Phi\right)$ can be extended to $\mathcal{A}$, the ring generated by $\mathcal{I}$. Hence for every $A \in \mathcal{A}$,

$$
-s\left(-x^{*}, Q\right) \lambda(A) \leq s\left(x^{*}, \Phi(A)\right) \leq \lambda(A) s\left(x^{*}, Q\right) .
$$

Consequently $\left|s\left(x^{*}, \Phi(A)\right)\right| \leq\left|s\left(x^{*}, Q\right)\right| \lambda(A)+\left|s\left(-x^{*}, Q\right)\right| \lambda(A)$. Since $A \mapsto$ $\lambda(A) s\left(x^{*}, Q\right)$ is $\sigma$-additive on $\mathcal{A}$ and bounded, $s\left(x^{*}, \Phi(\cdot)\right)$ can be extended to a measure $\mu_{x^{*}}: \sigma(\mathcal{A}) \rightarrow \mathbb{R}$, where $\sigma(A)$ consists of all Borel subsets of $[0,1]$.

Now let $B \in \sigma(\mathcal{A})$ and consider a sequence $\left(A_{n}\right)_{n} \subset \mathcal{A}$ such that $\lambda\left(B \triangle A_{n}\right) \rightarrow 0$. We prove that $\left(\Phi\left(A_{n}\right)\right)_{n}$ is a Cauchy sequence in $\left(c k(X), d_{H}\right)$.

In fact, for every natural numbers $n, m$, we have

$$
\begin{aligned}
& d_{H}\left(\Phi\left(A_{n}\right), \Phi\left(A_{m}\right)\right) \\
& =\sup _{x^{*} \in B\left(X^{*}\right)}\left|s\left(x^{*}, \Phi\left(A_{n}\right)\right)-s\left(x^{*}, \Phi\left(A_{m}\right)\right)\right| \\
& =\sup _{x^{*} \in B\left(X^{*}\right)}\left|s\left(x^{*}, \Phi\left(A_{n} \backslash A_{m}\right)\right)-s\left(x^{*}, \Phi\left(A_{m} \backslash A_{n}\right)\right)\right| \\
& \leq \sup _{x^{*} \in B\left(X^{*}\right)}\left|s\left(x^{*}, \Phi\left(A_{n} \backslash A_{m}\right)\right)\right|+\sup _{x^{*} \in B\left(X^{*}\right)}\left|s\left(x^{*}, \Phi\left(A_{m} \backslash A_{n}\right)\right)\right| \\
& \leq 2 \sup _{x^{*} \in B\left(X^{*}\right)}\left|s\left(x^{*}, Q\right)\right| \lambda\left(A_{n} \backslash A_{m}\right)+2 \sup _{x^{*} \in B\left(X^{*}\right)}\left|s\left(x^{*}, Q\right)\right| \lambda\left(A_{m} \backslash A_{n}\right) \\
& =k \lambda\left(A_{n} \backslash A_{m}\right)+k \lambda\left(A_{m} \backslash A_{n}\right) \\
& =k \lambda\left(A_{n} \triangle A_{m}\right),
\end{aligned}
$$

where $k=2\|Q\|$. Since $\lambda\left(A_{n} \triangle A_{m}\right) \rightarrow 0$, also $d_{H}\left(\Phi\left(A_{n}\right), \Phi\left(A_{m}\right)\right) \rightarrow 0$. Since $\left(c k(X), d_{H}\right)$ is a complete metric space, we obtain that $\left(\Phi\left(A_{n}\right)\right)_{n}$ is $d_{H^{-}}$convergent to an element of $c k(X)$.

At this point let us define $M(B):=\left(d_{H}\right) \lim _{n} \Phi\left(A_{n}\right)$ for $B \in \sigma(\mathcal{A})$. The multifunction $M$ is well defined. In fact, if $\left(A_{n}^{\prime}\right) \subset \mathcal{A}$ is another sequence such that $\lambda\left(A_{n}^{\prime} \triangle B\right) \rightarrow 0$, then also $\lambda\left(A_{n}^{\prime} \triangle A_{n}\right) \rightarrow 0$. Consequently,

$$
d_{H}\left(\Phi\left(A_{n}^{\prime}\right), \Phi\left(A_{n}\right)\right) \leq k \lambda\left(A_{n}^{\prime} \triangle A_{n}\right) \rightarrow 0 .
$$

Thus $\left(d_{H}\right) \lim _{n} \Phi_{n}\left(A_{n}^{\prime}\right)=\left(d_{H}\right) \lim _{n} \Phi_{n}\left(A_{n}\right)$. Moreover, $M$ is $c k(X)$-valued and is an extension of $\Phi$ to $\sigma(\mathcal{A})$.

We claim that $s\left(x^{*}, M\right)=\mu_{x^{*}}$ for every $x^{*} \in X^{*}$. In fact, let fix $x^{*} \in X^{*}$. It follows from the definition of $M$ that, for every $B \in \sigma(\mathcal{A}), s\left(x^{*}, \Phi\left(A_{n}\right)\right) \rightarrow$ $s\left(x^{*}, M(B)\right)$, where $\left(A_{n}\right)_{n}$ is one of the above considerated sequence.

On the other hand, $\left|\mu_{x^{*}}(B)-s\left(x^{*}, \Phi\left(A_{n}\right)\right)\right|=\left|\mu_{x^{*}}(B)-\mu_{x^{*}}\left(A_{n}\right)\right|=$ $\left|\mu_{x^{*}}\left(B \backslash A_{n}\right)-\mu_{x^{*}}\left(A_{n} \backslash B\right)\right| \leq\left|\mu_{x^{*}}\left(B \backslash A_{n}\right)\right|+\left|\mu_{x^{*}}\left(A_{n} \backslash B\right)\right| \leq k \lambda\left(B \triangle A_{n}\right) \rightarrow 0$, 
for every $B \in \sigma(\mathcal{A})$. Hence $s\left(x^{*}, M(B)\right)=\mu_{x^{*}}(B)$ for every $B \in \sigma(\mathcal{A})$. Therefore for each $x^{*} \in X^{*} s\left(x^{*}, M\right)$ is a measure. Since $M$ is $c k(X)$-valued, we have that $M$ is a multimeasure (see [14, Theorem 8.4.10]).

Finally for each $B \in \sigma(\mathcal{A})$ and each $x^{*} \in X^{*}$

$$
s\left(x^{*}, M(B)\right)=\mu_{x^{*}}(B) \leq s\left(x^{*}, Q\right) \lambda(B)=s\left(x^{*}, \lambda(B) Q\right) .
$$

Therefore $M(B) \subseteq \lambda(B) Q$ for each $B \in \sigma(\mathcal{A})$.

The following result improves [5, Theorem 3.1] valid for dominated convex compact valued multimeasures that can be representated by Pettis integrable multifunctions. More precisely we show that a Pettis integrable density can be obtained even considering dominated interval multimeasures.

Theorem 4.2. Let $\Phi: \mathcal{I} \rightarrow c k(X)$ be an interval multimeasure such that there exists a set $Q \in \operatorname{ck}(X)$ with $\Phi(I) \subseteq|I| Q$ for every $I \in \mathcal{I}$. Then there exists a multifunction $F:[0,1] \rightarrow c k(X)$ Pettis integrable in $c k(X)$ such that:

1. for every finitely additive selection $\phi$ of $\Phi$ there exists a Pettis integrable selection $f$ of $F$ with $\phi(I)=(P) \int_{I} f(t) d t$ for all $I \in \mathcal{I}$;

2. $\Phi(I)=(P) \int_{I} F d \lambda$ for all $I \in \mathcal{I}$.

Proof. By Proposition 4.1, $\Phi$ can be extended to a multimeasure $M: \sigma(\mathcal{A}) \rightarrow$ $c k(X)$ such that $M(B) \subseteq \lambda(B) Q$ for every $B \in \sigma(\mathcal{A})$. Therefore by of $[5$, Theorem 3.1] there exists a Pettis integrable multifunction $F:[0,1] \rightarrow c k(X)$ such that

1. for each countably additive selection $m$ of $M$, there exists a Pettis integrable selection $f$ of $F$ with $m(B)=(P) \int_{B} f d \lambda$, for each $B \in \sigma(\mathcal{A})$,

2. $M(B)=(P) \int_{B} F d \lambda$

We conclude that $F$ satisfies the required properties.

One can assume on Theorem 4.2 that for each $t \in[0,1]$ there exist $Q_{t} \in$ $c k(X)$ and $\delta_{t}>0$ such that $\Phi(I) \subseteq Q_{t}|I|$ for every interval $I$ containing $t$ with $|I|<\delta_{t}$. But a simple topological argument shows that these assumptions imply that $[0,1]$ is a finite union of non-overlapping closed intervals in each of which the assumptions of Theorem 4.2 are fulfilled.

Proposition 4.3. Let $\Phi: \mathcal{I} \rightarrow c k(X)$ be an interval multimeasure such that $V_{\Phi} \ll \lambda$. Assume that there exists a sequence $\left(I_{n}\right)_{n}$ of non-overlapping intervals such that $\lambda\left([0,1] \backslash \bigcup_{n} I_{n}\right)=0$ and for each natural number $n$ there exists a compact set $Q_{n} \subset X$ with the property that $\Phi(I) \subseteq|I| Q_{n}$ for all subinterval $I$ of $I_{n}$.

Then $\Phi$ is the primitive of a ck(X)-valued multifunction HKP-integrable in $c k(X)$. 
Proof. By Theorem 4.2, for each $n$ there exists a multifunction $G_{n}: I_{n} \rightarrow c k(X)$, Pettis integrable in $c k(X)$, such that

$$
\Phi(I)=(P) \int_{I} G_{n} d \lambda, \quad \text { for each interval } I \subseteq I_{n} .
$$

Let us consider now the multifunction $G:[0,1] \rightarrow c k(X)$ defined as $G(t):=$ $\sum_{n} G_{n}(t)$.

Since $V_{\Phi} \ll \lambda$, we have also $V_{s\left(x^{*}, \Phi\right)} \ll \lambda$ for every $x^{*} \in X^{*}$. Therefore by $\left[3\right.$, Theorem 3] for every $x^{*} \in X^{*}$ there exists $g_{x^{*}} \in H K([0,1])$ such that

$$
s\left(x^{*}, \Phi(I)\right)=(H K) \int_{I} g_{x^{*}} d \lambda, \quad \text { for all } I \in \mathcal{I} .
$$

Fix $x^{*} \in X^{*}$. For each $n$ and each interval $I \subset I_{n}$, equality (1) holds. But for the same $n$ and $I$ we have also

$$
s\left(x^{*}, \Phi(I)\right)=(H K) \int_{I} s\left(x^{*}, G_{n}\right) d \lambda .
$$

Therefore we obtain $(H K) \int_{I} s\left(x^{*}, G_{n}\right) d \lambda=(H K) \int_{I} g_{x^{*}} d \lambda$ for each $n$ and each interval $I \subset I_{n}$. It follows by [10, Theorem 9.12] that for every $n, s\left(x^{*}, G_{n}\right)=g_{x^{*}}$ almost everywhere on $I_{n}$ (and the exceptional set depends only on $x^{*}$ ).

By the definition of $G$ we have that $s\left(x^{*}, G\right)=g_{x^{*}}$ almost everywhere on $[0,1]$ (and the exceptional set depends only on $x^{*}$ ). Therefore, by [10, Theorem 9.10] $s\left(x^{*}, G\right)$ is $H K$-integrable. Since $x^{*}$ is arbitrary, then $G$ is scalarly $H K$-integrable.

Finally, if $I \in \mathcal{I}$ and $x^{*} \in X^{*}$, we have

$$
s\left(x^{*}, \Phi(I)\right)=(H K) \int_{I} g_{x^{*}} d \lambda=(H K) \int_{I} s\left(x^{*}, G\right) d \lambda .
$$

We conclude that $G$ is $H K P$-integrable in $c k(X)$ and that $\Phi$ is its $H K P$ primitive.

4.2. The $c w k(X)$ case. Now we are going to consider the more general case of $c w k(X)$-valued multifunctions.

Proposition 4.4. Let $\Phi: \mathcal{I} \rightarrow \operatorname{cwk}(X)$ be an interval multimeasure such that $V_{\Phi} \ll \lambda$. Assume that $s\left(x^{*}, \Phi(I)\right) \geq 0$ for every $x^{*} \in X^{*}$ and for every $I \in \mathcal{I}$. Then $\Phi$ can be extended to a $\sigma$-additive multimeasure $M: \mathcal{L} \rightarrow c w k(X)$ of $\sigma$-finite variation and with $M \ll \lambda$.

Proof. Since $V_{\Phi} \ll \lambda$, we have also $V_{s\left(x^{*}, \Phi\right)} \ll \lambda$ for each $x^{*} \in X^{*}$. By [3, Theorem 3], for every $x^{*} \in X^{*}$ there exists $g_{x^{*}} \in H K([0,1])$ such that

$$
s\left(x^{*}, \Phi(I)\right)=(H K) \int_{I} g_{x^{*}} d \lambda, \quad \text { for every } I \in \mathcal{I} .
$$


Since $s\left(x^{*}, \Phi\right) \geq 0$, it follows by [10, Theorem 9.12] that $g_{x^{*}} \geq 0$ almost everywhere on $[0,1]$. By [10, Theorem 9.13], $g_{x^{*}}$ is Lebesgue integrable for every $x^{*} \in X^{*}$. Moreover, $V_{s\left(x^{*}, \Phi\right)}$ is a measure over all Borel sets of $[0,1]$. By $[9$, Theorem 2], $V_{s\left(x^{*}, \Phi\right)}(B)=\int_{B} g_{x^{*}} d \lambda$ for every $B \in \sigma(\mathcal{A})$. Now let us consider the family

$$
\mathcal{B}:=\left\{B \in \sigma(\mathcal{A}): \exists C_{B} \in \operatorname{cwk}(X) \mid \forall x^{*} \in X^{*}, s\left(x^{*}, C_{B}\right)=\int_{B} g_{x^{*}} d \lambda\right\} .
$$

Notice that for every $B \in \mathcal{B}, s\left(x^{*}, C_{B}\right) \leq \int_{0}^{1} g_{x^{*}} d \lambda=s\left(x^{*}, \Phi([0,1])\right)$ for every $x^{*} \in X^{*}$. Hence $C_{B} \subseteq \Phi([0,1])$ for every $B \in \mathcal{B}$.

It is clear that $\mathcal{B}$ contains $\mathcal{A}$. We claim that $\mathcal{B}$ is a monotone class. In fact, let $\left(B_{n}\right)_{n}$ be a monotone increasing sequence of $\mathcal{B}$ and let $C_{B_{n}} \in \operatorname{cwk}(X)$ such that $s\left(x^{*}, C_{B_{n}}\right)=\int_{B_{n}} g_{x^{*}} d \lambda$ for every $x^{*} \in X^{*}$. By the Monotone Convergence Theorem (see [10, Theorem 3.21]) $\lim _{n} \int_{B_{n}} g_{x^{*}} d \lambda=\int_{\cup_{n} B_{n}} g_{x^{*}} d \lambda$. Moreover, also $\left(C_{B_{n}}\right)_{n}$ is a monotone increasing sequence, in fact, for every $n$ and every $x^{*} \in X^{*}, s\left(x^{*}, C_{B_{n}}\right)=\int_{B_{n}} g_{x^{*}} d \lambda \leq \int_{B_{n+1}} g_{x^{*}} d \lambda=s\left(x^{*}, C_{B_{n+1}}\right)$. Hence $C_{B_{n}} \subseteq C_{B_{n+1}}$ for every $n$.

Consequently, $\lim _{n} s\left(x^{*}, C_{B_{n}}\right)=s\left(x^{*}, \bigcup_{n} C_{B_{n}}\right)=s\left(x^{*}, \overline{\bigcup_{n} C_{B_{n}}}\right)$. In fact, the first equality follows from the fact that $\lim _{n} s\left(x^{*}, C_{B_{n}}\right)=\sup _{n} s\left(x^{*}, C_{B_{n}}\right)=$ $s\left(x^{*}, \bigcup_{n} C_{B_{n}}\right)$, the second equality is a property of the support function. Since $\bigcup_{n} C_{B_{n}} \subseteq \Phi([0,1]) \in c w k(X)$, we have $\overline{\bigcup_{n} C_{B_{n}}} \in c w k(X)$. Hence we have that $s\left(x^{*}, \overline{\bigcup_{n} C_{B_{n}}}\right)=\int_{\bigcup_{n} B_{n}} g_{x^{*}} d \lambda$ and therefore $\bigcup_{n} B_{n} \in \mathcal{B}$.

Let $\left(B_{n}\right)_{n}$ be a monotone decreasing sequence of $\mathcal{B}$, and let $C_{B_{n}} \in \operatorname{cwk}(X)$ such that $s\left(x^{*}, C_{B_{n}}\right)=\int_{B_{n}} g_{x^{*}} d \lambda$ for every $x^{*} \in X^{*}$. Clearly $\lim _{n} \int_{B_{n}} g_{x^{*}} d \lambda=$ $\int_{\bigcap_{n} B_{n}} g_{x^{*}} d \lambda$. Moreover, also $\left(C_{B_{n}}\right)_{n}$ is a monotone decreasing sequence. Thus $\lim _{n} s\left(x^{*}, C_{B_{n}}\right)=s\left(x^{*}, \bigcap_{n} C_{B_{n}}\right)=s\left(x^{*}, \overline{\bigcap_{n} C_{B_{n}}}\right)$. Moreover, we have that $\overline{\bigcap_{n} C_{B_{n}}} \in c w k(X)$, because $\bigcap_{n} C_{B_{n}} \subseteq \Phi([0,1]) \in c w k(X)$. Hence we have that $s\left(x^{*}, \overline{\bigcap_{n} C_{B_{n}}}\right)=\int_{\bigcap_{n} B_{n}} g_{x^{*}} d \lambda$ for every $x^{*} \in X^{*}$. Therefore $\bigcap_{n} B_{n} \in \mathcal{B}$.

By the Monotone Class Theorem (see [11]), $\mathcal{B}$ contains the smallest $\sigma$ algebra containing $\mathcal{A}$. Hence $\mathcal{B}=\sigma(\mathcal{A})$.

Let us define $M: \sigma(\mathcal{A}) \rightarrow c w k(X)$ as follows: $M(B)=C_{B}, B \in \sigma(\mathcal{A}) . M$ is a multimeasure, because for every $x^{*} \in X^{*}, s\left(x^{*}, M(\cdot)\right)$ is a Lebesgue integral. Since $M$ is $c w k(X)$-valued, $M$ is also a $d_{H}$-multimeasure.

We prove that $M \ll \lambda$. In fact, if $B \in \sigma(\mathcal{A})$ and $\lambda(B)=0$, then for every $x^{*} \in X^{*}, s\left(x^{*}, M(B)\right)=\int_{B} g_{x^{*}} d \lambda=0$. Consequently, $\|M(B)\|=$ $\sup _{x^{*} \in B\left(X^{*}\right)}\left|s\left(x^{*}, M(B)\right)\right|=0$, hence $M(B)=\{0\}$.

It remains to prove that $M$ is of $\sigma$-finite variation. Since $V_{\Phi} \ll \lambda$, we have that $V_{\Phi}$ is $\sigma$-finite. Let $\left(B_{n}\right)_{n} \subseteq \sigma(\mathcal{A})$ be a partition of $[0,1]$ such that $V_{\Phi}\left(B_{n}\right)<+\infty$ for every $n$. Fix $n$ and let $\left\{B_{n, 1}, \ldots, B_{n, k}\right\} \subseteq \sigma(\mathcal{A})$ be a partition of $B_{n}$. Then for every $x^{*} \in B\left(X^{*}\right)$ and every $j=1, \ldots, k$ we obtain $s\left(x^{*}, M\left(B_{n, j}\right)\right)=V_{s\left(x^{*}, \Phi\right)}\left(B_{n, j}\right) \leq V_{\Phi}\left(B_{n, j}\right)$. Hence for every $j=1, \ldots, k$, 
$\left\|M\left(B_{n, j}\right)\right\| \leq V_{\Phi}\left(B_{n, j}\right)$ and therefore $\sum_{j=1}^{k}\left\|M\left(B_{n, j}\right)\right\| \leq V_{\Phi}\left(B_{n}\right)$. Finally, $|M|\left(B_{n}\right) \leq V_{\Phi}\left(B_{n}\right)<+\infty$.

Since $M \ll \lambda$, we can extend $M$ to $\mathcal{L}$, because any measurable set is the union of a Borel set and a set of zero Lebesgue measure. The proof is complete.

Remark 4.5. The assumption $s\left(x^{*}, \Phi(I)\right) \geq 0$ for every $x^{*} \in X^{*}$ and every $I \in \mathcal{I}$, implies that $0 \in \Phi(I)$ for every $I \in \mathcal{I}$.

It is worth to observe that without this assumption, Proposition 4.4 fails even if $X$ is the real line. A simple example can be made by taking a HenstockKurzweil but not Lebesgue integrable real valued function $g$ and setting $f=g \cdot x_{0}$, where $X \ni x_{0} \neq 0$. Then $\varphi(I):=(H K) \int_{I} f d \lambda$ is the required counterexample.

Theorem 4.6. Let $X$ be a Banach space with the $R N P$ and let $\Phi: \mathcal{I} \rightarrow \operatorname{cwk}(X)$ be an interval multimeasure such that $V_{\Phi} \ll \lambda$. Then $\Phi$ admits a cbc $(X)$-valued density $F$ which is HKP-integrable in cwk $(X)$.

Proof. Let us consider first the case when $s\left(x^{*}, \Phi\right) \geq 0$ for every $x^{*} \in X^{*}$. By Proposition 4.4, $\Phi$ can be extended to a $\sigma$-additive multimeasure $M: \mathcal{L} \rightarrow$ $\operatorname{cwk}(X)$ such that $M$ is of $\sigma$-finite variation and $M \ll \lambda$.

Let $\left(A_{n}\right)_{n}$ be a sequence of pairwise disjoint sets of $\mathcal{L}$ such that $\bigcup_{n} A_{n}=$ $[0,1]$ and $|M|\left(A_{n}\right)<+\infty$ for all $n$. Let us denote by $M_{n}$ the restriction of $M$ to all measurable subsets of $A_{n}$. Each $M_{n}$ is a $c w k(X)$-valued (hence $c b c(X)$ valued) multimeasure of finite variation. Moreover, since $M \ll \lambda$, also $M_{n} \ll \lambda$, for all $n$.

Since $X$ has the RNP, by [5, Theorem 4.1] we have that, for all $n, M_{n}$ has a density $F_{n}: A_{n} \rightarrow c b c(X)$ which is Pettis integrable in $c b c(X)$.

Now let us define the multifunction $F:[0,1] \rightarrow c b c(X)$ as follows:

$$
F(t):=F_{n}(t), \quad \text { if } t \in A_{n} .
$$

We check that $F$ is scalarly integrable. Let us fix $x^{*} \in X^{*}$. Since $M$ is $c w k(X)$ valued, $s\left(x^{*}, M\right)$ is a positive (by construction) real-valued measure absolutely continuous with respect to $\lambda$. Therefore by the classic Radon-Nikodým Theorem, there exists $h_{x^{*}} \in L^{1}([0,1])$ such that

$$
s\left(x^{*}, M(A)\right)=\int_{A} h_{x^{*}} d \lambda, \quad \text { for every } A \in \mathcal{L} .
$$

Moreover, for each $n, F_{n}$ is a Pettis integrable density of $M_{n}$, hence

$$
s\left(x^{*}, M_{n}(A)\right)=\int_{A} s\left(x^{*}, F_{n}\right) d \lambda, \quad \text { for every } A \in \mathcal{L}, A \subseteq A_{n} .
$$


It follows that for every $n, s\left(x^{*}, F_{n}\right)=h_{x^{*}}$ almost everywhere on $A_{n}$ (and the exceptional set depends only on $\left.x^{*}\right)$.

By the definition of $F$ we have also that $s\left(x^{*}, F\right)=h_{x^{*}}$ (and the exceptional set depends only on $\left.x^{*}\right)$. Therefore $s\left(x^{*}, F\right)$ is integrable. Since $x^{*}$ is arbitrary, then $F$ is scalarly integrable.

Finally we observe that for every $A \in \mathcal{L}$ and every $x^{*} \in X^{*}$,

$$
s\left(x^{*}, M(A)\right)=\int_{A} h_{x^{*}} d \lambda=\int_{A} s\left(x^{*}, F\right) d \lambda .
$$

Therefore $F$ is a Pettis integrable (in $c w k(X))$ density of $M$. In particular,

$$
\Phi(I)=(P) \int_{I} F d \lambda, \quad \text { for every } I \in \mathcal{I} .
$$

In the general case, let $\phi$ be a finitely additive selection of $\Phi$ (existing by Proposition 3.5) and let consider $\Psi:=\Phi-\phi$. It is clear that $s\left(x^{*}, \Psi\right) \geq 0$ for every $x^{*} \in X^{*}$. We have also that $V_{\Psi} \ll \lambda$, since $V_{\Phi} \ll \lambda$ and $V_{\phi} \ll \lambda$. Consequently, $\Psi$ has a density $G:[0,1] \rightarrow c b c(X)$ Pettis integrable in $c w k(X)$. Since $X$ has the RNP, by $[2$, Theorem 3.6] $\phi$ has a variationally Henstock integrable (and then Henstock integrable) density $f:[0,1] \rightarrow X$ (see $[2,21]$ for the definition of variational Henstock integral).

Now let us consider the multifunction $F:=G+f$. Clearly $F$ is $c b c(X)$ valued. Moreover, $s\left(x^{*}, F\right)=s\left(x^{*}, G\right)+\left\langle x^{*}, f\right\rangle$, for every $x^{*} \in X^{*}$. Since each $s\left(x^{*}, G\right)$ is Lebesgue integrable and each $\left\langle x^{*}, f\right\rangle$ is $H K$-integrable, also $s\left(x^{*}, F\right)$ is $H K$-integrable. Hence $F$ is scalarly $H K$-integrable.

Finally, for every $x^{*} \in X^{*}$ and for every $I \in \mathcal{I}$, we have

$$
\begin{aligned}
s\left(x^{*}, \Phi(I)\right) & =s\left(x^{*}, \Psi(I)\right)+\left\langle x^{*}, \phi(I)\right\rangle \\
& =\int_{I} s\left(x^{*}, G\right) d \lambda+(H K) \int_{I}\left\langle x^{*}, f\right\rangle d \lambda \\
& =(H K) \int_{I} s\left(x^{*}, F\right) d \lambda .
\end{aligned}
$$

We conclude that $F$ is $H K P$-integrable in $\operatorname{cbc}(X)$ and $\Phi(I)=(H K P) \int_{I} F d \lambda$, for every $I \in \mathcal{I}$.

Remark 4.7. In general, under the hypothesis of Theorem 4.6, the density of $\Phi$ is only $c b c(X)$ and not $c w k(X)$-valued, as the following example shows (see [6, Exemple 2]).

Let $X$ be the space $l_{1}$ and let $\left(e_{n}\right)_{n \geq 0}$ be the canonical base of $l_{1}$. Let $\left(\alpha_{n}^{k}\right)_{n, k \geq 0}$ be a sequence of real numbers such that

$$
\sum_{n \geq 0}\left|\alpha_{n}^{k}\right|=1 \text { for every } k \geq 0 \text { and } \sum_{k \geq 0}\left(\sum_{n \geq 0}\left|\alpha_{n}^{k}\right|^{2}\right)^{\frac{1}{2}}<+\infty .
$$


Let $\left(r_{n}\right)_{n \geq 0}$ be the sequence of the Rademacher functions. For $k \geq 0$ and $t \in[0,1]$, set $\sigma_{k}(t):=\left(\alpha_{n}^{k} r_{n}(t)\right)_{n \geq 0} \in l_{1}$. Now let define $F(t):=\overline{c o}\left\{\sigma_{k}(t): k \geq 0\right\}$, $t \in[0,1]$. Then, the $c b c\left(l_{1}\right)$-valued multifunction $F$ is Pettis integrable in $c w k\left(l_{1}\right)$, but $F(t) \notin c w k\left(l_{1}\right)$ a.e.

Remark 4.8. If the Banach space is the real line, then $c b c(\mathbb{R})=c k(\mathbb{R})=$ $c w k(\mathbb{R})$ and the Henstock integrability coincides with the Henstock-KurzweilPettis integrability. Let us give in such a case a simple proof of Theorem 4.6, using properties of the real line.

Proof of Theorem 4.6 for $X=\mathbb{R}$. Since $\Phi$ is $c k(\mathbb{R})$-valued, the set $\Phi(I)$ is a closed bounded interval of the real line for all $I \in \mathcal{I}$.

Let us consider the real functions $\varphi, \psi: \mathcal{I} \rightarrow \mathbb{R}$ defined respectively by $\varphi(I):=\min \Phi(I)$ and $\psi(I):=\max \Phi(I)$.

Of course, $\varphi$ and $\psi$ are selections of $\Phi$. Since $V_{\Phi} \ll \lambda$, we have $V_{\varphi} \ll \lambda$ and $V_{\psi} \ll \lambda$. So by [2, Theorem 3.6], $\varphi$ and $\psi$ are differentiable almost everywhere in $[0,1]$ and there exist $f, g \in \mathcal{H} \mathcal{K}([0,1])$ such that $\varphi(I)=(H K) \int_{I} f d \lambda$ and $\psi(I)=(H K) \int_{I} g d \lambda$ for each $I \in \mathcal{I}$. Moreover, $\varphi^{\prime}=f$ and $\psi^{\prime}=g$ a.e.

Since $\varphi \leq \psi$, we have $(H K) \int_{I} f d \lambda \leq(H K) \int_{I} g d \lambda$ for all $I \in \mathcal{I}$. Consequently, $f \leq g$ a.e.

Now let consider the multifunction $F$ defined by

$$
F(t):= \begin{cases}{[f(t), g(t)]} & \text { if } f(t) \leq g(t) \\ \{0\} & \text { elsewhere. }\end{cases}
$$

It is clear that $F$ is $c k(\mathbb{R})$-valued. Let $\gamma$ be a selection of $\Phi$. Since $V_{\Phi} \ll \lambda$, also $V_{\gamma} \ll \lambda$. Therefore by [3, Theorem 3], $\gamma$ is differentiable almost everywhere in $[0,1]$ and there exists $h \in \mathcal{H} \mathcal{K}([0,1])$ such that $\gamma(I)=(H K) \int_{I} h d \lambda$. Moreover, $\gamma^{\prime}=h$ a.e.

Since $\varphi \leq \gamma \leq \psi$, then we get also that $f \leq h \leq g$ a.e. Consequently, $h(t) \in F(t)$ for almost every $t \in[0,1]$. So, changing eventually the values in a negligible set, we have that $h$ is a selection of $F$.

Since $f, g \in \mathcal{H} \mathcal{K}([0,1])$, for each $\varepsilon>0$, there exists a gauge $\delta$ on $[0,1]$ such that

$$
\left|(H K) \int_{0}^{1} f d \lambda-\sum_{j=1}^{p} f\left(t_{j}\right)\right| I_{j}||<\frac{\varepsilon}{2} \quad \text { and } \quad\left|(H K) \int_{0}^{1} g d \lambda-\sum_{j=1}^{p} g\left(t_{j}\right)\right| I_{j}||<\frac{\varepsilon}{2},
$$

for every $\delta$-fine partition $\left\{\left(I_{j}, t_{j}\right)\right\}_{j=1}^{p}$ of $[0,1]$. Thus 


$$
\begin{aligned}
& d_{H}\left(\Phi([0,1]), \sum_{j=1}^{p} F\left(t_{j}\right)\left|I_{j}\right|\right) \\
& =d_{H}\left(\left[(H K) \int_{0}^{1} f d \lambda,(H K) \int_{0}^{1} g d \lambda\right],\left[\sum_{j=1}^{p} f\left(t_{j}\right)\left|I_{j}\right|, \sum_{j=1}^{p} g\left(t_{j}\right)\left|I_{j}\right|\right]\right) \\
& \leq\left|(H K) \int_{0}^{1} f d \lambda-\sum_{j=1}^{p} f\left(t_{j}\right)\right| I_{j}||+\left|(H K) \int_{0}^{1} g d \lambda-\sum_{j=1}^{p} g\left(t_{j}\right)\right| I_{j}|| \\
& <\varepsilon
\end{aligned}
$$

for every $\delta$-fine partition $\left\{\left(I_{j}, t_{j}\right)\right\}_{j=1}^{p}$ of $[0,1]$. Therefore $F$ is Henstock integrable and $(H) \int_{0}^{1} F d \lambda=\Phi([0,1])$.

Finally, using Hausdorff distance we infer for each $I \in \mathcal{I}$,

$d_{H}\left(\Phi(I),(H) \int_{I} F d \lambda\right) \leq\left|\varphi(I)-(H K) \int_{I} f d \lambda\right|+\left|\psi(I)-(H K) \int_{I} g d \lambda\right|=0$.

Hence $\Phi(I)=(H) \int_{I} F d \lambda$ for every $I \in \mathcal{I}$ and the proof is over.

In [22], the following result has been proved.

Theorem 4.9. Let $X$ be a separable Banach space with the RNP. Assume that also $X^{*}$ has the RNP. Let $M$ be a cwk $(X)$-valued multimeasure of $\sigma$-finite variation and such that $M \ll \lambda$. Then $M$ admits a unique density $F:[0,1] \rightarrow$ $\operatorname{cwk}(X)$ which is Pettis integrable in cwk $(X)$.

Under the same assumptions for the Banach space $X$, by previous theorem we have the following result for interval multimeasures.

Theorem 4.10. Let $X$ be a separable Banach space with the RNP. Assume that also $X^{*}$ has the $R N P$. Let $\Phi: \mathcal{I} \rightarrow \operatorname{cwk}(X)$ be an interval multimeasure such that $V_{\Phi} \ll \lambda$. Then $\Phi$ admits a cwk $(X)$-valued density $F$ which is HKPintegrable in cwk(X).

Proof. First let us consider the particular case when $s\left(x^{*}, \Phi\right) \geq 0$ for every $x^{*} \in X^{*}$. By Proposition 4.4, $\Phi$ can be extended to a $\sigma$-additive multimeasure $M: \mathcal{L} \rightarrow \operatorname{cwk}(X)$ such that $M$ is of $\sigma$-finite variation and $M \ll \lambda$. By hypothesis, $X$ is separable, has the RNP and also its dual $X^{*}$ has the RNP. Therefore by Theorem $4.9, M$ has a density $F:[0,1] \rightarrow c w k(X)$ which is Pettis integrable in $c w k(X)$. Consequently, we have

$$
\Phi(I)=(P) \int_{I} F d \lambda, \quad \text { for every } I \in \mathcal{I} .
$$


In the general case, let $\phi$ be a finitely additive selection of $\Phi$ and let consider $\Psi:=\Phi-\phi$. It is clear that $s\left(x^{*}, \Psi\right) \geq 0$ for every $x^{*} \in X^{*}$. We have also that $V_{\Psi} \ll \lambda$, since $V_{\Phi} \ll \lambda$ and $V_{\phi} \ll \lambda$. Consequently, $\Psi$ has a density $G:[0,1] \rightarrow c w k(X)$ Pettis integrable in $c w k(X)$. By [2, Theorem 3.6] $\phi$ has a variationally Henstock (then a Henstock) integrable density $f:[0,1] \rightarrow X$ (see $[2,21]$ for the definition of variational Henstock integral).

Now let consider the multifunction $F:=G+f$. Clearly $F$ is $c w k(X)$-valued. Moreover, it is easy to check that $s\left(x^{*}, F\right)=s\left(x^{*}, G\right)+\left\langle x^{*}, f\right\rangle$, for every $x^{*} \in X^{*}$. Since each $s\left(x^{*}, G\right)$ is Lebesgue integrable and each $\left\langle x^{*}, f\right\rangle$ is $H K$-integrable, also $s\left(x^{*}, F\right)$ is $H K$-integrable. Hence $F$ is scalarly $H K$-integrable.

Finally for every $x^{*} \in X^{*}$ we have

$$
\begin{aligned}
s\left(x^{*}, \Phi(I)\right) & =s\left(x^{*}, \Psi(I)\right)+\left\langle x^{*}, \phi(I)\right\rangle \\
& =\int_{I} s\left(x^{*}, G\right) d \lambda+(H K) \int_{I}\left\langle x^{*}, f\right\rangle d \lambda \\
& =(H K) \int_{I} s\left(x^{*}, F\right) d \lambda,
\end{aligned}
$$

for every $I \in \mathcal{I}$. We conclude that $F$ is $H K P$-integrable in $c w k(X)$ and

$$
\Phi(I)=(H K P) \int_{I} F d \lambda, \quad \text { for every } I \in \mathcal{I} .
$$

Acknowledgement. The authors are grateful to the anonymous reviewers for their valuable remarks.

\section{References}

[1] Artstein, Z., Set-valued measures. Trans. Amer. Math. Soc. 165 (1972), $103-125$.

[2] Bongiorno, B., Di Piazza, L. and Musiał, K., A variational Henstock integral characterization of the Radon-Nikodym property. Illinois J. Math. 53 (2009)(1), $87-99$.

[3] Bongiorno, B., Di Piazza, L. and Skvortsov, V., A new full descriptive characterization of Denjoy-Perron integral. Real Anal. Exchange 21 (1995/96), $256-263$.

[4] Bourgin, R., Geometric Aspects of Convex Sets with the Radon-Nikodym Property. Lect. Notes Math. 993. Berlin: Springer 1983.

[5] Cascales, B., Kadets, V. and Rodríguez, J., Radon-Nikodým theorems for multimeasures in non-separable spaces. Zh. Mat. Fiz. Anal. Geom. 9 (2013)(1), $7-24$.

[6] Costé, A., La propriété de Radon-Nikodým en intégration multivoque (in French). C. R. Acad. Sci. Paris Sér. A-B 280 (1975)(22), Aii, A1515-A1518. 
[7] Costé, A. and Pallu de la Barrière, R., Radon-Nikodým theorems for set-valued measures whose values are convex and closed. Comment. Math. Prace Mat. 20 $(1977 / 78)(2), 283-309$.

[8] Diestel, J. and Uhl, J. J., Vector Measures. Math. Surweys 15. Providence (RI): Amer. Math. Soc. 1977.

[9] Di Piazza, L., Variational measures in the theory of the integration in $R^{m}$. Czechoslovak Math. J. 51 (2001)(1), 95 - 110.

[10] Gordon, R. A., The Integrals of Lebesgue, Denjoy, Perron and Henstock. Grad. Stud. Math. 4. Providence (RI): Amer. Math. Soc. 1994.

[11] Halmos, P. R., Measure Theory. New York: Springer 1974.

[12] Henstock, R., Theory of Integration. London: Butterworths 1963.

[13] Hiai, F., Radon-Nikodým theorems for set-valued measures. J. Multivariate Anal. 8 (1978)(1), 96 - 118.

[14] Hu, S. and Papageorgiou, N. S., Handbook of Multivalued Analysis. Vol. I: Theory. Dordrecht: Kluwer Acad. Publ. 1997.

[15] Kurzweil, J., Generalized ordinary differential equations and continuous dependence on a parameter. Czechoslovak Math. J. 7 (1957), 418 - 446.

[16] Martellotti, A., Musiał, K. and Sambucini, A. R., A Radon-Nikodým theorem for the Bartle-Dunford-Schwartz integral with respect to finitely additive measures. Atti Sem. Mat. Fis. Univ. Modena 42 (1994), 625 - 633.

[17] Martellotti, A. and Sambucini, A. R., A Radon-Nikodým theorem for multimeasures. Atti Sem. Mat. Fis. Univ. Modena 42 (1994), 579 - 599.

[18] Musiał, K., Topics in the theory of Pettis integration (School of Measure Theory and Real Analysis Grado (Italy) 1991). Rend. Istit. Mat. Univ. Trieste 23 (1991)(1), $177-262$.

[19] Musiał, K., Pettis Integrability of multifunctions with values in arbitrary Banach spaces. J. Convex Anal. 18 (2011)(3), 769 - 810.

[20] Sambucini, A. R., Un teorema di Radon-Nikodym in spazi localmente convessi rispetto all'integrazione per seminorme (in Italian). Riv. Mat. Univ. Parma 5 (1995)(4), $49-60$.

[21] Schwabik, S. and Ye, G., Topics in Banach Space Integration. Ser. Real Anal. 10. Hackensack (NJ): World Sci. Publ. 2005.

[22] Ziat, H., Approximation and decomposability in the space of Pettis integrable functions. Int. J. Math. Anal. 6 (2012)(31), $1519-1537$.

Received June 12, 2014; revised October 30, 2014 